\title{
INVESTIGAÇÃO TEÓRICA DAS PROPRIEDADES MECÂNICAS E OPTOELETRÔNICAS DOS MATERIAIS SBMO3 (M = B, AL E GA)
}

\author{
Carlos Rodolfo Bomfim Lopes Souza ${ }^{1}$; Nilton Souza Dantas ${ }^{2}$ \\ 1. Bolsista PIBIC/Fapesb, Graduando em bacharelado em Física, Universidade Estadual de Feira de Santana, e-mail: \\ crblsouza@gmail.com \\ 2. Orientador, Departamento de Ciências Exata, Universidade Estadual de Feira de Santana, e-mail: \\ nilton.dantas@gmail.com
}

PALAVRAS-CHAVE: Teoria do funcional da densidade; propriedades ópticas; função dielétrica.

\section{INTRODUÇÃO}

Os novos materiais funcionais, assim chamados porque não se encontram na natureza e são obtidos por técnicas de engenharia de materiais, possuem propriedades com as mais diversas aplicações na indústria optoeletrônica, mecânica, de energia, dentre outras. Um grupo desses novos materiais é o das perovskitas, as quais são de ocorrência rara na natureza, mas que recentemente têm despertado o interesse de diversos pesquisadores devido a diversas propriedades de interesse prático, tais como termoeletricidade, piezeletricidade, ferroeletricidade, (anti-)ferromagnetismo, transição metal-isolante, (Zubko, Gariglio, Gabay, et al, 2012) (Kimura, Goto, Shintani, Ishizaka, Arima, Tokura, 2003) (Hur, Park, Sharma, Ahn, Guha, Cheong, 2004), dentre outras.

No grupo de perovskitas do tipo $\mathrm{AMO}_{3}$, um material que tem sido bem estudado é o $\mathrm{BiMO}_{3}$ (Behram et al, 2016). Os materiais formados por elementos de uma mesma coluna da tabela periódica costumam ter propriedades químicas semelhantes, o que sugere que seja possível formar um grupo de perovskitas do tipo $\mathrm{AMO}_{3}$ com os elementos das colunas III-V, onde A é um elemento da coluna V e M um elemento da coluna III. Alguns materiais desse grupo são $\mathrm{SbMO}_{3}$, modelado a partir do $\mathrm{BiMO}_{3}$ pela substituição do átomo de Bi por Sb.

Nesse trabalho, foram conduzidos estudos teóricos através da modelagem computacional do cristal cúbico para os materiais $\mathrm{SbMO}_{3}(\mathrm{M}=\mathrm{B}$, $\mathrm{Al}$ e $\mathrm{Ga})$ com o objetivo de caracterizá-los com suas propriedades eletrônicas, ópticas e estruturais.

Aplicando a Teoria do Funcional da Densidade, tal como está implementada no pacote computacional WIEN2k (Blaha, Schwarz, Madsen, Kvasnicka, Luitz, 2002), foi possível calcular a energia de formação do cristal e, a partir daí todas as propriedades de interesse: foi obtida a densidade de estados, a estrutura de bandas, o GAP óptico, a função dielétrica e as constantes elásticas.

\section{MATERIAL E MÉTODOS OU METODOLOGIA (ou equivalente)}

No desenvolvimento desse trabalho foi usado o software WIEN2k, instalado no cluster do laboratório de novos materiais do Departamento de Ciências Exatas. Para alcançar os objetivos, o trabalho foi conduzido na seguinte metodologia:

1 - Inicialmente, foi construída uma célula unitária cúbica para cada material e logo após, foram feitos ajustes dos parâmetros de rede através da minimização da energia de formação de cada célula unitária;

2 - A seguir foram obtidas as estruturas de bandas de energia para todos os materiais;

3 - O próximo passo foi o cálculo das densidades de estados;

4 - Foi obtida a função dielétrica para cada material;

5 - Foi feito o cálculo das constantes elásticas para cada material;

RESULTADOS E/OU DISCUSSÃO (ou Análise e discussão dos resultados)

O valor do parâmetro de rede otimizado de cada material encontra-se na Tab. 1 . 
Tabela 1: Parâmetro de rede de cada célula.

$\mathrm{SbMO}_{3}(\mathrm{M}=\mathrm{B}, \mathrm{Al}, \mathrm{Ga})$

B

Al

Ga
Parâmetro de rede (Å)

6,669

7,106

7,323

Percebe-se que há um aumento aproximadamente linear do volume da célula com a substituição do elemento central por outro de maior número atômico.

Com o parâmetro otimizado, foi obtida a estrutura de bandas de energia e a densidade

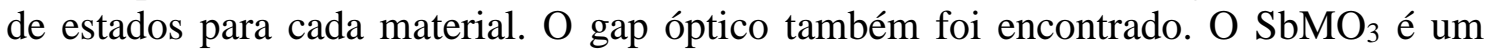
material que apresenta gap direto, na direção do ponto X. Há uma diminuição do gap com a substituição do átomo central por outro de maior número atômico. Como encontrado na literatura, a inserção de átomos de maior número atômico em cristais tende a diminuir o gap óptico (Dantas et al, 2008) (Silva, 2004). Isso é mostrado nos gráficos da estrutura de bandas.

Tabela 2: gap óptico dos materiais

$\mathrm{SbMO}_{3}(\mathrm{M}=\mathrm{B}, \mathrm{Al}, \mathrm{Ga}) \quad$ Gap óptico (eV)

B

Al

Ga

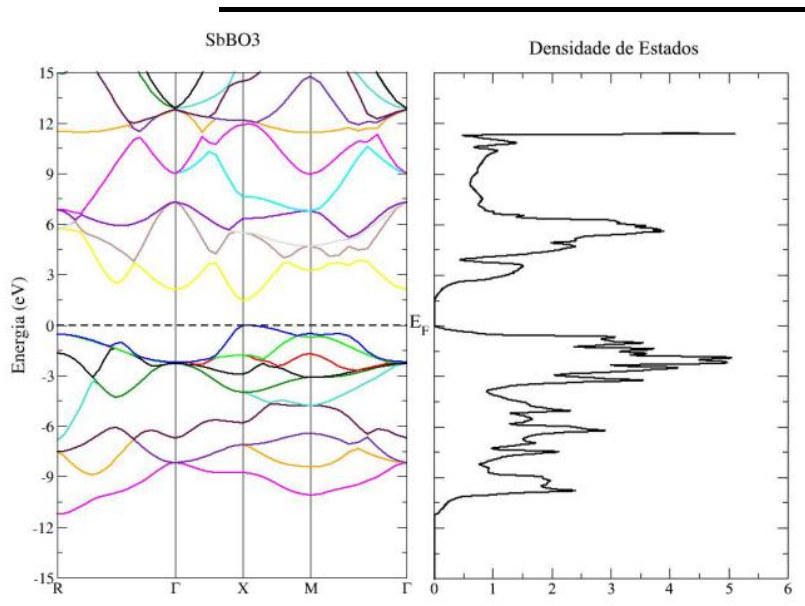

(a)
1,436

1,166

1,004
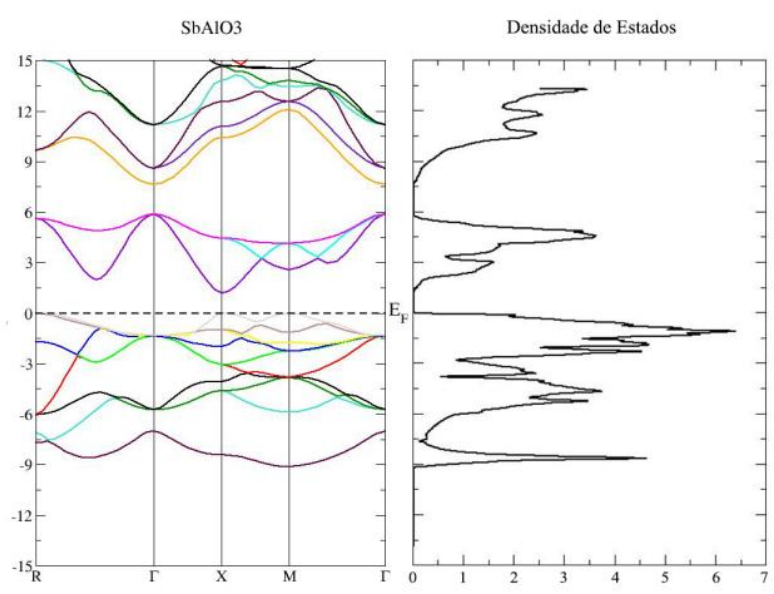

(b)

Figura 1: estrutura de bandas para: (a) $\mathrm{SbBO} 3$ e (b) SbAlO3.

A diminuição do gap óptico ocorre devido à introdução de novos estados da banda $\mathrm{p}$ próximo ao nível de Fermi, que é formada na ligação entre $\mathrm{Sb}^{+3}$ e $\left[\mathrm{MO}_{3}\right]^{-3}$.

As propriedades ópticas dos materiais podem ser descritas pela função dielétrica, $\varepsilon(\omega)=\varepsilon_{1}(\omega)+i \varepsilon_{2}(\omega)$, que representa como o material responde a aplicação de uma onda eletromagnética de comprimento de onda $\lambda>>a$, onde $a$ é o parâmetro de rede do material. A parte imaginária da função dielétrica é mostrada nos gráficos da Fig. 2. Através das relações de Kramers-Kronig, a parte real pode ser encontrada (Klingshirn, 2005). 


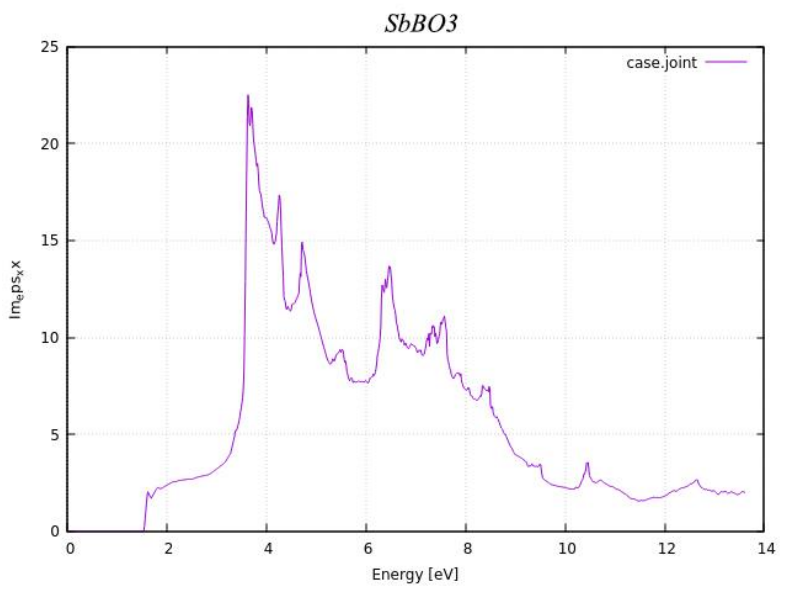

(a)

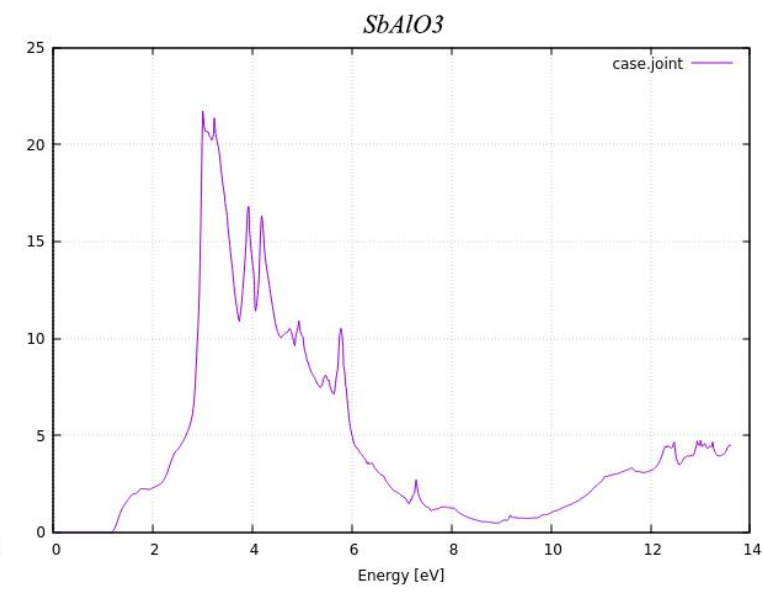

(b)

Figura 2: Parte imaginária da função dielétrica para (a) $\mathrm{SbBO}$ e (b) $\mathrm{SbAlO3}$

Os picos de absorção na faixa entre 3 a 6 eV em $\mathrm{SbBO}_{3}, \mathrm{SbAlO}_{3}$ e $\mathrm{SbGaO}_{3}$ e são devido às transições entre as bandas $\mathrm{s}$ e $\mathrm{p}$ do $\mathrm{Sb}$ e $\mathrm{O}$. Isso pode ser explicado através das densidades de estados parciais. A maior densidade de estados é encontrada nas faixas de 1,5 a 6 eV nas bandas s e p do $\mathrm{Sb}$ e de -3 a 0 eV na banda $\mathrm{p}$ do $\mathrm{O}$.

Observa-se que há um deslocamento do pico de absorção para regiões de menores energias quando o átomo central é substituído por átomos de maior número atômico. Esse deslocamento evidencia o fechamento do gap e, portanto, da diferença de energia entre as transições eletrônicas. As constantes elásticas e o bulk modulus foram calculados através de pequenas deformações no cristal. A tabela 2 apresenta as constantes $\mathrm{c}_{11}, \mathrm{c}_{12}$ e $\mathrm{c}_{44} \mathrm{e} \mathrm{o}$ valor do bulk modulus.

Tabela 3: constantes elásticas e bulk modulus dos materiais

\begin{tabular}{ccccc}
\hline SbMO $_{3}$ (M=B, Al, Ga) & C11 (GPa) & C12 (GPa) & C44 (GPa) & B0 (GPa) \\
B & 212.345 & 236.0614 & 222.9276 & 228.1551 \\
Al & 270.3529 & 144.0623 & 123.7645 & 186.1590 \\
Ga & 368.6116 & 98.0308 & 90.2106 & 188.2244 \\
\hline
\end{tabular}

\section{CONSIDERAÇÕES FINAIS (ou Conclusão)}

Neste trabalho foi feito a investigação teórica utilizando métodos em primeiros princípios das propriedades optoeletrônicas e mecânicas de uma perovskita de fórmula $\mathrm{SbMO}_{3}, \mathrm{M}=\mathrm{B}$, Al e Ga. Foi utilizada a DFT e o método LAPW para solução das equações de Kohn-Sham e o funcional de troca-correlação PBE-GGA, tal como implementada no pacote WIEN2k.

Os resultados mostram que o parâmetro de cresce à medida em que o átomo central é substituído por outro de número atômico maior. Esse incremento de volume já era esperado, pois com o aumento do raio iônico do átomo central, há o aumento das distâncias das ligações químicas, o que acarreta na formação de células maiores.

Os resultados obtidos sugerem que essa perovskita é um sólido semicondutor se $M=B$, $\mathrm{Al}$ e Ga. O gap direto do material indica que pode ser um material promissor para aplicações optoeletrônicas e fabricação de células fotovoltaicas. A diminuição do gap ocorre devido à contribuição da banda $\mathrm{p}$ do $\left[\mathrm{MO}_{3}\right]^{-3}$, que introduz novos estados na região de 0 a $6 \mathrm{eV}$. A contribuição dessas bandas também causa o deslocamento das faixas de absorção da função dielétrica para regiões de menores energia. 
Esses resultados indicam que se pode controlar o gap e a banda de absorção de fótons do material trocando-se o átomo central.

\section{REFERÊNCIAS}

SCHWARZ, Karlheinz; BLAHA, Peter; MADSEN, G. K. H. Electronic structure calculations of solids using the WIEN2k package for material sciences. Computer Physics Communications, v. 147, n. 1-2, p. 71-76, 2002.

T. KIMURA, T. GOTO, H. SHINTANI, K. ISHIZAKA, T. ARIMA, Y. TOKURA, Nature 426 (2003) 55.

N. HUR, S. PARK, P.A. SHARMA, J.S. AHN, S. GUHA, S.-W. CHEONG, Nature 429 (2004) 392.

KLINGSHIRN, Claus F. Semiconductor optics. Springer Science \& Business Media, 2005.

SILVA, A. F. da et al , Proc. of Int. Bulk Nitride Semicond. (Krakow, 2004), J. Cryst. Growth 281, 151

(2005).

DANTAS, N. S. et al, App. Phys. Lett.,Vol. 92, 121914, (2008).

P. ZUBKO, S. GARIGLIO, M. GABAY, et al., Annu. Rev. Condens. Matter Phys. 2 (2012) 141.

R. B. BEHRAM et al, Material Science in Semiconductor Precessing, 41 (2016) 297. 